\title{
COORDINATED DATA ANALYSIS: A NEW METHOD FOR THE STUDY OF PERSONALITY AND HEALTH*
}

PREPRINT

\author{
Sara J. Weston \\ Department of Psychology \\ University of Oregon \\ Eugene, OR, USA \\ weston.sara@gmail.com
}

\author{
Eileen K. Graham \\ Department of Medical Social Sciences \\ Northwestern University \\ Chicago, IL, USA \\ eileen.graham@northwestern.edu
}

\author{
Andrea M. Piccinin \\ Department of Psychology \\ University of Victoria \\ Victoria, British Columbia, Canada \\ piccinin@uvic.ca
}

August 30, 2019

\begin{abstract}
A majority of research by personality psychologists examining health has utilized publicly available datasets, for good reason. These resources are often the only available datasets large enough to detect expected effect sizes and may contain biological or genetic data that is difficult to obtain. However, researchers tend to examine only one large dataset at a time. Given recent meta-research on the robustness and replicability of "established" findings, all researchers should take greater care to evaluate the evidentiary value of their findings and seek methods to increase their robustness. Personality and aging psychologists who use publicly available datasets have a unique tool at their disposal in order to achieve this goal, namely, more publicly available datasets. More specifically, psychologists may use coordinated analysis (Hofer and Piccinin. 2009; Piccinin and Hofer, 2008) to examine relationships across several large datasets and, using the tools of meta-analysis, identify generalizable effect sizes and examine heterogeneity across countries and methods. This chapter describes the motivation for coordinated analysis, the process of using this method, and details several examples.
\end{abstract}

The proliferation of panel studies and other publicly-available datasets has greatly advanced the study of healthy aging, as these datasets serve as a low-burden avenue for examining longitudinal relationships, often including real-world outcomes. However, as the Open Science Reform Movement has brought attention to common pitfalls in data analysis (Munafò et al., 2017), it is clear that careful steps must be taken to ensure robust and rigorous analyses of panel study data. The current chapter will review the benefits and pitfalls of panel studies and then discuss one method of rigorous data analysis: the coordinated analysis.

\section{The benefits of panel studies}

The nature of psycho-social change being one of the primary interests of developmental and aging researchers (Baltes and Nesselroade, 1979), panel studies and other similar publicly-available datasets are widely used in psychology. Panel studies - the repeated assessment of large samples across years or decades - allow for estimations of change in psycho-social constructs across developmental periods and after major life events (Vartanian, 2010). More than just being longitudinal in nature, panel studies lend several advantages to a research project.

${ }^{*}$ This chapter will appear in the forthcoming handbook Personality and Healthy Aging in Adulthood, edited by Patrick L. Hill and Mathias Allemand and published by Springer Nature. 
First, many are large in size (i.e., assess many individuals), allowing for the estimation of small but meaningful effect sizes. For example, the Midlife in the United States Study (MIDUS, Brim et al., 1999) has been used to link sense of purpose to net worth (Hill et al., 2016). The effect size estimated was small (with a standardized regression coefficient of about 0.04) but translates into a large real-world effect (for every standard deviation increase in purpose, participants had on average $\$ 20 \mathrm{~K}$ greater net worth). Large samples also allow for better group comparisons. The relationship of personality to health, for instance, can differ by ethnicity; large samples, like in the Hawaii Personality and Health Cohort (Hampson and Goldberg, 2006), allow for the estimation of these marginal effects without undue loss of statistical power (McClelland and Judd, 1993).

As a second advantage, panel studies are often well-funded and well-staffed, allowing for the collection of data that would otherwise be too expensive or time-consuming to collect. The UK Biobank (Sudlow et al. 2015), for instance, has genetic data on more than half a million participants to date. The Health and Retirement Study has biomarker and prescription drug use data on thousands of its participants. Individual research labs, even teams of labs, would struggle to find the money and manpower to assess genetics or biomarkers in samples of this magnitude; to then link such data to decades worth of survey research is almost unthinkable.

Which brings us to the third advantage of panel studies: time and money. Accessing a panel study is often free or, if not, less costly than paying for the cost of interviewing and assessing thousands of participants. This is especially useful for researchers with limited financial resources, such as early career researchers - students, post-docs, untenured faculty - and for researchers who work in teaching-focused environments without grant funding. Moreover, developmental and lifespan questions often require years, even decades, of data collection, making such research difficult for anyone. Moreover, many research questions about the lifespan will need a lifespan's worth of data.

\section{Challenges in analyzing panel studies}

Analysis of panel studies and other publicly available datasets is not without pitfalls. We have named several panel studies already in this chapter (e.g., the UK Health and Lifestyle Survey, the MIDUS, the UK Biobank). While each has been used to address similar questions, the published results are not directly comparable. For example, each has been used to assess the relationship of neuroticism to health. Neuroticism is related to greater mortality in the UK Health and Lifestyle Survey (Shipley et al., 2007). In the MIDUS, neuroticism was unrelated to mortality risk (Turiano et al., 2015). Finally, using the UK Biobank, Gale et al. (2017) found that neuroticism was associated with a reduction in mortality risk. But these results are not directly comparable. Each panel study used a different measure of neuroticism - the first and third used the Eysenck Personality Inventory with a binary response choice, while the second used the MIDI with a 4-point Likert scale. Each study used different covariates: while all controlled for age and gender, the second also controlled for race, marital status and education; the third study included at least a dozen additional covariates. The time between personality assessment and mortality varied across studies: 21 years, 11 years, and 14 years, respectively. And each study used their own approach to variable transformation and standardization. There are other differences of course, including the location of the study, ages of the participants at baseline, year of data collection, etc. It is clear to see why comparing these findings cannot clarify the link between neuroticism and mortality. There are too many differences between the samples and analyses to make sense of why any differences in results emerge.

Adjusting for these differences is difficult under the best of circumstances; however, the scale of panel studies allows for model overfitting, which can bias published results. By overfitting, we mean mistakenly fitting a model to samplespecific noise (for a discussion, see Yarkoni and Westfall, 2017). Overfit models are problematic as they can include spurious relationships that will not generalize outside of the sample at hand. One of the easiest ways to overfit a model is to include too many terms, such as too many covariates or too many polynomials and interactions. Panel studies can include thousands of variables and tens of thousands of subjects; motivated researchers have the opportunity to fit many different models, on different subsets of the data. These models may ultimately be over-complex, over-fitted and p-hacked. P-hacking, or modifying analyses with the goal of obtaining a p-value smaller than the significance threshold (Simmons et al. 2011), is often assumed to be an intentional act of the researcher, but even honest researchers can unknowingly engage in this practice. For example, researchers can bias results if they have previous knowledge of a dataset; given that researchers often return to the same panel studies multiple times, this is not only possible but likely (see Weston et al. 2019b). Even without deliberate p-hacking, prior knowledge of datasets precludes the traditional use of p-values, which can only be interpreted under the assumption that the statistical test is independent of any other statistical test; prior knowledge violates this assumption.

Unfortunately, our modern system of publishing research relies heavily on the use of statistical significance. Therefore, researchers who wish to publish secondary data analysis must still use statistical significance, despite the violation of the independent test assumption. Responsible researchers will seek additional ways to improve the robustness of such tests. Luckily, there is a proliferation of statistical tools for such tasks. Machine learning techniques can be used to evaluate the robustness of effect sizes (Yarkoni and Westfall, 2017). The use of preregistration and other open science practices 
can effectively limit researcher degrees of freedom and enhance transparency (Weston et al., 2019b). Researchers who use publicly available panel studies have a particularly useful tool: more panel studies. In other words, researchers can leverage the availability of large datasets to answer simple but important research questions, limit researcher degrees of freedom, and test moderators imposed by time, location and study design. This process is referred to as coordinated analysis.

\section{Coordinated analysis}

A coordinated analysis is the analysis of multiple independent datasets in a way that optimizes the comparison of results (Hofer and Piccinin, 2009). The primary goal of a coordinated analysis is to maximize the opportunities for direct comparisons of results. To achieve this goal, researchers analyze identical (or nearly identical) statistical models across multiple datasets but preserve other differences in data (e.g., measurement instrument, cohort). These meaningful differences allow researchers to estimate the generalizability of an effect across time, culture and instrument or, in the case where differences emerge, identify potential moderators of an effect. Often, the results from individual datasets are compared using the tools of meta-analysis, which allow for statistical estimating of heterogeneity and the moderating effect of sample-level characteristics, such as location or year of data collection.

Coordinated analysis is a subset of a larger group of analytic tools, referred to as integrative data analysis. A coordinated analysis can be collaborative if using proprietary datasets or if seeking to involve many researchers; it may also be an independent exercise if a researcher limits himself or herself to only directly accessible datasets.

\section{Recommendations for Coordinated Analysis}

The steps of coordinated data analysis follow much the same process as the scientific method: a research question is answered by testing specific hypotheses with data. This process quickly becomes complex, as these steps are simultaneously implemented in many different datasets and then integrated into a concise, if not simple, picture. Consequently, researchers should be careful to limit the complexity of their hypotheses and statistical models. Moreover, coordinated analyses often become team efforts, especially if using proprietary data. The authors of this chapter have each participated in coordinated analyses conducted by over two dozen researchers at a time. While the logistics of coordinating analyses across many datasets (and in some cases, several continents) is made easier by the Internet, we note that ensuring all researchers are on the same page is no simple task. It is with this in mind that we make the following recommendations:

\section{Study simple yet foundational relationships}

Simple models are the key to successful coordinated analyses, for two reasons. First, overly complex models are difficult to integrate across multiple datasets. For example, structural equation models often require modification to converge and adequately fit one dataset; attempting to fit an identical model across multiple datasets with adequate fit may be a Sisyphean task. Second, overly complex models limit the number of datasets eligible for inclusion in a coordinated analysis, as these models include more variables, sometimes requiring multiple measurement occasions with specific temporal ordering. Inclusion of an extra covariate in a linear model can reduce the datasets in which the full model can be estimated. We recommend that researchers limit their hypotheses to variables deemed essential and to answer questions using the simplest form of the model possible.

When developing a research question, researchers should consider that coordinated analysis is most useful for establishing foundational relationships or processes and estimating the generalizability of those key effects. For example, personality psychologists are interested in the relationship between trait conscientiousness and mortality; cognitive psychologists are interested in the growth and decline in working memory over time. Findings such as these are central to forming theories and studying complex processes. Therefore, in order to best develop new theories, psychologists must establish the existence of such relationships, the size of the relationship, and the conditions under which the association is strongest. Coordinated analysis provides an efficient way to estimate all three of these key characteristics. We note that, while this method may be used for exploratory research as well, it is not well-suited for such analyses given the time and planning required; as we will discuss below, coordinated analyses often include extensive coordination with multiple team members, many analytic decisions prior to analysis, and the development and testing of analytic scripts prior to data analysis. These processes are facilitated by clear research aims and specific, confirmatory hypotheses. In other words, the labor involved in a coordinated analysis can make it difficult to fully explore a series of datasets, though it can facilitate the generation of new hypotheses for current or subsequent projects (Hofer and Piccinin, 2009. 2010; Piccinin et al. 2013). 


\section{Establish minimum inclusion criteria}

Once the analytic model is specified, it becomes necessary to identify the criteria a particular dataset must meet for the model to be tested within that dataset and to be included in a coordinated analysis. Inclusion criteria may include the following: constructs measured, measurement instrument and quality, number and spacing of measurement occasions, populations sampled, and data quality checks. Ideally, simple hypotheses and simple models require fewer inclusion criteria. For example, a simple correlation test requires that a dataset measure only two constructs. It is important to consider the conditions under which a dataset is included or excluded from a coordinated analysis before beginning the task of gathering and organizing data. Doing so not only saves time, but reduces the risk of post-hoc changes.

The choice of inclusion criteria ultimately dictates the scope of the coordinated analysis, the ability to compare results, and the estimation of generalizability of findings. More stringent criteria - e.g., all datasets must use the same instrument to measure a construct of interest - allows for easier comparison of results across samples, but limits the generalizability of results to only that instrument. On the other hand, criteria regarding the quality of data, such as internal reliability cutoffs or necessary base rates for binary variables, reduces the noise in estimates. We recommend that researchers limit variable inclusion criteria to only those necessary to adequately fit the model. Doing so ensures the quality of results while allowing for the maximum number of datasets. For example, (Graham et al. 2019a) examined trajectories of Big Five personality traits using multi-level growth models in a coordinate analysis framework. The minimum requirement for a dataset to be included was three measurement occasions of at least one of the Big Five personality traits. Studies with a single trait were still included in the project and simply excluded from any analysis for which they did not have the appropriate data.

Once minimum inclusion criteria are defined, researchers must comprehensively search for available datasets. There are a number of excellent resources available for identifying appropriate datasets for a given project. Many data repositories have archived publicly available data so that anyone can register and access them. For example, ICPSR houses data on social research (broadly speaking) and contains a number of specialized "collections" of datasets (e.g. the Resource Center for Minority Data [RCMD], and the National Archive of Computerized Data on Aging [NACDA]). The National Institute for Mental Health (NIMH) has a similarly organized repository, containing specialized collections of archived mental health data, sorted by subject area (e.g. the Osteoarthritis Initiative, the National Database for Autism Research). There are also a number of international data networks, such as Ageing Trajectories of Health: Longitudinal Opportunities and Synergies (ATHLOS). Many of the datasets in the above-mentioned repositories are publicly available.

However, limiting oneself only to public data could introduce new bias into the results of a project. Therefore, we recommend also exploring access to non-public data as well. The Integrative Analysis of Longitudinal Studies of Aging and Dementia (IALSA) is one such network. The IALSA is a network of studies of aging from around the world, many of which are not publicly accessible for legal or ethical reasons. The network has created a pipeline by which individual researchers can use these data for their research purposes while not accessing the data itself. This is achieved through the use of developing and sharing analytic scripts. An individual researcher who is interested in conducting a coordinated analysis will write an analytic script (for example, an R script) that details precisely which analyses will be run; with what options, assumptions, or conditions; and in what order. The analytic script may also include essential transformations of variables, creations of indices, and post-hoc follow-up tests or data-visualization. Importantly, these scripts are written to work on any dataset with the appropriate variables (more information on scripting is including in the next section). This script is shared with a data-analyst associated with the dataset of interest. The analyst runs the analysis script on their data and sends the resulting output back to the individual researcher who can incorporate the output into a meta-analysis and write-up of results. The IALSA website, currently powered by Maelstrom, contains a variable search tool that can be used to identify studies with the variables/constructs needed. Contact information for each study is provided if an IALSA-affiliated study described in the Maelstrom catalogue is a good fit for a given project. The IALSA network and its website should be cited/acknowledged in any product arising from knowledge gained from the site.

\section{Leverage scripting technology}

While scripting languages are not new to statistical analysis, they have increased in appeal during the renewed focus on reproducibility in the social sciences. As a consequence, it is easier to not only write analytic scripts but share them through online platforms (e.g., the Open Science Framework or GitHub) and collaboratively develop them. We therefore recommend that researchers conducting coordinated analyses rely heavily on scripting languages. The benefits of using this method include (1) ensuring that models are specified identically across datasets, (2) the capacity to immediately summarize and integrate results, and (3) the integration of results directly into the manuscript. 
Regarding ensuring identical specification, coordinated analyses are meant to examine the heterogeneity of a particular relationship across multiple samples, measurement instruments, and/or time points. Therefore, other sources of variability should be avoided at all costs. One potential source of variability is the use of different analyses across datasets. For a solo researcher conducting all analyses, this means care should be taken to ensure that the models estimated on each dataset are identical, including decision rules surrounding the identification and removal of outliers, the use of different underlying estimation procedures, when and how to transform variables, the process for dealing with missing data, and the modeling of dependencies between family members, to name a few. While the most conscientious researchers among us will clearly document every single analytic decision and check that each decision was applied consistently across datasets, there is still room for human error if the analyst repeats every step by hand. For a team of data analysts, the potential for discrepancies increases exponentially, as data analysts may vary in their expertise or experience with the analysis at hand, their opinions and standard operating procedures for the analysis, and even the statistical software they use to estimate models, given that different softwares often have different default settings. For example, the default ANOVA sums of squares calculation for SPSS is Type III, but for R is Type I. Without scripting technology, coordination could only be achieved by detailed and specific communication of analytic decisions among these analysts and the appropriate implementation of these decisions by each analyst.

However, if each dataset is analyzed using an identical script, then consistency across analyses is more likely, regardless of the carefulness of a researcher or the communication skills of a team. An effective analytic script employs logical statements to ensure that a dataset meets the minimum inclusion criteria, checks that data are properly cleaned and coded, identifies which variables are available and thus which models can be tested, estimates the appropriate models, extracts descriptive and inferential statistics, and saves the information necessary for writing the final manuscript and estimating overall (meta-analytic) effects, if those are to be included. In other words, an effective analytic script can be run by any data analyst on any dataset without requiring idiosyncratic changes. This of course requires additional effort upfront. Scripts should be tested on simulated data to ensure they work properly. But this effort ultimately pays off when it comes time to analyze many datasets simultaneously. Not only does this step in a coordinated analysis go quickly, but there are fewer opportunities for human error. An identical script ensures identical analyses across studies.

Scripting technology also facilitates the summary and integration of results across datasets. Analytic scripts can be used to identify, extract, and name key statistics in a systematic fashion, allowing for those key statistics to be efficiently integrated into a summary. For example, imagine conducting a coordinated analysis on the correlation between two variables. The analytic script used on each dataset could calculate and generate a name that identifies the statistic and the dataset, e.g., $r_{-} A, r_{-} B, r_{-} C$ for datasets $\mathrm{A}, \mathrm{B}$, and $\mathrm{C}$. It would then be easy to identify all statistics that include the prefix " $r_{-}$" and combine these into an average correlation. In fact, such scripts can be written prior to analyses conducted on individual datasets, meaning a researcher conducting a coordinated analysis can easily re-estimate her meta-analytic effects as new data come in or after mistakes are identified and fixed.

Finally, there have been recent advances in integrating open source statistical software, like R, with open source text formatting software, like LaTex. In other words, it is becoming easier and easier to automatically populate the text of a manuscript with statistics generated through $\mathrm{R}$ and to incorporate and even reference summary tables and figures. These integrations both increase the efficiency with which researchers can summarize and communicate results and also reduce human error in the generation of figures and tables.

\section{Preregister analyses prior to data cleaning}

Preregistration, the act of describing in detail the analysis plan for a study prior to working with data, benefits a project on many fronts. These benefits are not limited to coordinated analysis, but apply to all research. Preregistration was developed to increase transparency and reduce researcher degrees of freedom. By making analytic decisions prior to viewing results, researchers can avoid making data-dependent decisions, which violate assumptions of null hypothesis significance testing and invalidate the traditional interpretations of results (Gelman and Loken 2013). Simply put, if a researcher sees a relationship in a dataset and then statistically tests that relationship, the test is no longer a test; this is analogous to flipping the coin and then calling heads or tails.

Similarly, preregistration can be used to designate which parts of a project are confirmatory tests and which are exploratory. Preregistration does not preclude the inclusion of additional analyses; rather, a preregistration makes clear which analyses were planned prior to data analysis and which were developed after having seen data. This distinction is crucial, as analytic choices informed by data violate the assumption of independence that is central to probability theory and hypothesis testing. For this reason, preregistration provides a tool for responding to reviewers who ask for more complex analyses in order to "find" a result they are satisfied with. Specifically, if researchers believe they must run additional analyses to be published, they can include those analyses to satisfy reviewers, but use the preregistration to justify caution in interpreting results and labeling them as exploratory. Publicly posting 
preregistrations with date-stamps counters the file-drawer problem, as a record of the study is available, even if the results are not published.

In our own work, we have found that preregistration is especially useful for coordinated analyses. The act of completing a preregistration, especially one that is detailed and thorough, helps to organize the process going forward. For example, preregistration forms may ask a researcher to outline a plan to enact when outliers are detected; this should then prompt a researcher to write syntax that tests for outliers and carry out this plan when they are found. Overall, anticipating not only the primary model, but the conditions that must be met to run this model and the contingency plans when the model fails aids researchers in developing working syntax and limiting data-dependent decisions. In addition, given that coordinated analyses are often conducted by teams of researchers, a preregistration can help ensure all team members are on the same page concerning the analyses and interpretations. Writing a preregistration and giving all team members an opportunity to read, refine, and agree to the preregistration limits conflict at later stages in the project.

We feel strongly that robust and replicable research in lifespan development need to approach the analysis planning stage using theory-driven analysis decisions, not data-driven analysis decisions. The latter can easily lead to model-overfitting, which by extension leads to non-replicable results. Some longitudinal modeling techniques by definition require data-driven decisions: for example, in growth mixture modeling, the first step in the model is to identify a set of groups in a dataset, and the second set using those groups to predict an outcome. The results of these models tend to be highly specific to a given dataset and are often impossible to replicate. One solution is to use cross-validation techniques, where each dataset in the coordinated analysis is subset into a "training" sample and a "test" sample, where the data-driven portion of the analysis decision are fine-tuned and coordinated across studies within the training samples using cross-validation techniques, then ultimately evaluated based on performance in the hold-out test samples.

\section{Consider generalizability}

One of the strengths of coordinated analysis is the opportunity to formally test the generalizability of an effect across time, culture, population, and method. Establishing generalizability, or the limits thereof, is a key goal of research, as it can dramatically affect how scientists choose to study a phenomenon and the way they interpret results. If conscientiousness is associated with health only when researchers measure personality using a specific instrument, then we are less confident in this result. If extraversion follows the same developmental trajectory in all cultures, we are more confident. If cognitive ability predicts income in all cohorts except one, we look to historical events that may have interfered with this relationship. In short, coordinated analysis not only provides a high-powered and precise estimate of specific parameter; it can also test the degree to which that parameter estimate applies to the world population.

The tests of generalizability are only as good as the data. We therefore encourage researchers (1) seek datasets that collectively cover a range of cultures, timepoints, measurement instruments, or any variable which may affect the hypothesized relationship, and (2) examine as many moderating contexts as possible. The latter largely involves examining differences between different datasets: geographic location, cohort, measurement spacing, and instrument are all possible moderators of an effect. We recommend consulting Hofer and Piccinin (2009) for a discussion of common sources of between-study heterogeneity.

Estimating the degree of between-study heterogeneity is often a goal of meta-analysis, and so several metrics exist to serve this function. The I2 statistic (Higgins and Thompson. 2002) represents the proportion of variance in the estimates that is due to heterogeneity between the studies. While this statistic is commonly used, it is also subject to bias, as it assumes homogeneity of the within-study variances (which is almost never the case); other statistics, such as Q, estimate this heterogeneity with less bias (Crippa et al. 2016). These statistics are invariably accompanied by tests of significance, which may be used as a tool to avoid imprecise guessing or eyeballing of heterogeneity. We do not recommend one estimate over another but rather suggest researchers estimate several statistics and come to a more holistic conclusion. We also recommend estimating whether effect sizes can be predicted by study-level characteristics, such as year of study. We also note that consistency and generalizability can be tested within-study as well, if multiple measures of a construct are available, or if participants differ in age, gender, or other key characteristics. Include planned tests of heterogeneity between studies and tests of moderation within studies in a preregistration.

\section{Coordinated Analysis in Action}

In the following section, we illustrate the approach of coordinated analysis by detailing one a specific example of its use. We start by summarizing the motivation of the study, and then describe the application of our five recommendations. 


\section{Motivation}

The research project we describe here attempts to answer the following research question: Does the relationship between neuroticism and health depend on levels of conscientiousness? The relationship of neuroticism to health is less defined than many personality researchers may think. While typically neuroticism is associated with worse health - including greater engagement in unhealthy behaviors, increased risk of developing chronic conditions, and reduced lifespan there are enough published null and even positive associations between neuroticism and health to conclude that the relationship between neuroticism and health may be more complex. This heterogeneity was first described by Friedman (2000) who suggested that such diverse findings could result from individuals high in neuroticism falling into two categories: the traditional neurotics, who deal with stress through maladaptive coping behaviors like smoking and drinking, and the healthy neurotics, who proactively address changes in their health and, consequently, detect major health concerns at earlier and more treatable stages. Implicit in this theory is the presence of a moderator, or a third variable which determines whether a person high in neuroticism is healthy or unhealthy.

While the specific moderator of neuroticism was not identified through this theory - and indeed, several have been examined empirically, including SES (Elliot et al., 2016; Hagger-Johnson et al., 2012; Weston et al., 2018), physical health (Weston et al., 2018), and even self-rated health (Gale et al. |2017) - the most promising candidate moderator is trait conscientiousness. Conscientiousness, the tendency to be rule-following, industrious, and orderly, is commonly presented as the trait needed to focus the natural worries of a person high in neuroticism on controllable domains (like health) and address problems in this domain through adaptive and healthy behaviors. Often individuals high in both traits are described as likely being "vigilant" towards their health (Turiano et al., 2013, p. 87; Weston and Jackson. 2015. p. 7).

Since the introduction of the healthy neuroticism concept almost two decades ago, the idea is frequently found in theoretical work describing the relationship of personality to health (e.g., Friedman, 2019; Murray and Booth, 2015). Despite the popularity of this theory, the empirical evidence supporting this interaction is limited and mainly focused on smoking and alcohol consumption (c.f., Turiano et al., 2012; Vollrath and Torgersen, 2002). Sometimes, the interaction is only present in three-way interactions, such as the interaction of conscientiousness, neuroticism, and gender to predict mortality risk in Friedman et al. (2010), or in subsamples, such as only among individuals diagnosed with some chronic conditions in Weston and Jackson (2015). Moreover, a number of empirical studies show no relationship of this interaction to health behaviors (e.g., Atherton et al., 2014), adaptive responding to health news (Weston and Jackson, 2016), or more distal outcomes, such as frailty (Stephan et al. 2017). It is unclear whether these discrepant results are due to each of these analyses being underpowered to detect the interaction (McClelland and Judd. 1993), or if this interaction is only related to health among some populations or when measured using certain instruments, or if the true interaction effect is null and the published significant results are simply capturing spurious effects. What is needed to determine the true size of this interaction effect is a highly powered study that can examine meaningful differences between samples, namely, a coordinated analysis. We will now describe how we either followed the recommendations of this chapter to develop our coordinated analysis project or, when we did not follow our own advice, how this affected our project.

\section{Choosing simple yet foundational relationships}

When designing the coordinated analysis best suited to test whether the relationship of neuroticism to health was indeed moderated by conscientiousness, we first attempted to define the simplest relationship underlying this theory. This proved to be a difficult task, specifically because the empirical evidence that inspired the original description of healthy neuroticism (Friedman, 2000) was entirely based on discrepancies in the neuroticism-mortality association. However, the bulk of the published empirical evidence for this interaction examined health behaviors, most often smoking and alcohol consumption (e.g., Turiano et al., 2012), as these are fairly straightforward to assess and the more proximal associates with personality.

Choosing one outcome over another would impact the interpretation of the healthy neuroticism theory: examining mortality would be a direct test of the primary theory, that is, does conscientiousness help us to reconcile major differences in the relationship between neuroticism and mortality, but would remain silent on the processes underlying these differences. Examining behaviors, on the other hand, would help to clarify how neuroticism may be adaptive, but not whether those behaviors would have a substantial impact on one's survival. We chose to examine both mortality and behavior, specifically smoking, alcohol consumption, and physical activity. We also examined chronic condition status (hypertension, diabetes, and heart condition) for good measure. These outcomes were relegated to three separate but parallel projects, a coordinated coordinated-analysis, if you will. Analytic decisions were repeated across the three projects, to the extent possible. 
Ultimately, our analysis examines the interaction of neuroticism by conscientiousness on each outcome in question. We included several covariates that were known to be related to neuroticism and health or conscientiousness and health: age, reported gender, education level, and any other Big 5 personality traits (agreeableness, openness, extraversion) measured concurrently with neuroticism and conscientiousness. Importantly, each model was tested with and without covariates, to examine sensitivity to the inclusion of these additional variables.

We note here that the choice of seven outcome variables is not in line with the recommendation to keep analyses simple. However, given that these models were nearly identical across outcome type, we were able to conduct the analyses efficiently and also provide a clear synopsis of the study. Moreover, in tension between simplicity and thoroughness, we decided it was better, here, to err on the side of thoroughness, as this would best illuminate the boundaries of the healthy neuroticism effect.

\section{Establish minimum inclusion criteria}

We limited study selection to the IALSA network, which is sufficiently large to capture many populations at several time points with a variety of measurement instruments. Our inclusion criteria were broad: studies were included in the analyses if, during at least one wave of data collection, participants were measured on trait neuroticism, trait conscientiousness and at least one of the seven outcomes, in addition to age, gender, and education level. We identified 15 datasets that met these criteria, all of which had at least one health behavior and one chronic condition variable; 13 datasets included mortality information.

\section{Leverage scripting technology}

This coordinated analysis was the first, to our knowledge, to be conducted entirely using open-source scripting statistical software, in this case, using R. We are the first to acknowledge that using this software had enormous benefits, but also required an additional level of forethought in planning. Given the use of IALSA, many datasets in the current analysis are not generally shared outside the data collection organization. Instead, each organization associated with a dataset appointed a data analyst for the project, who was responsible for ensuring that data were cleaned properly and for analyzing the data according to our specifications. Over a dozen data analysts were involved in this project, plus additional team members who had collected data or were organizing members of the IALSA network. To facilitate analysis, we created a single script and shared it with all data analysts. Remarkably, data analysts were able to convene in a single location for several days, when they all ran these scripts simultaneously on data. Certainly, there were moments when new decisions had to be made, but they were easily communicated to the group, as every single analyst made the exact same change to the same location of a script. There was no concern that different softwares were using different estimation procedures. Moreover, even very junior scientists were able to participate, as they were not required to produce analytic scripts of their own but were able to simply use the script given to them, and this was reportedly a very memorable learning experience for these young scientists.

Because we used scripting technology, we were not only able to complete analyses on each individual dataset within a matter of hours, but it took only minutes to integrate these results into a series of overall weighted effects and display them using forest plots, or a graphical display of the estimates and confidence intervals of each individual dataset and the overall, weighted effect size and confidence interval (Lalkhen and McCluskey, 2008). The primary benefit of having written our integration scripts ahead of time was that it gave our team the opportunity to process results immediately and discuss the likelihood that this interaction sufficiently explained heterogeneity in the literature. Finally, we integrated our scripts into our manuscript using R and LaTex, which has allowed us to easily write up the results of our coordinated analysis and even make adjustments as analysts have identified mistakes in data cleaning.

\section{Preregister analysis prior to data cleaning}

For the healthy neuroticism project, we registered analyses on the Open Science Framework (osf.io/kcquy), which increased the confidence we have in interpreting our results. We anticipate that this preregistration will help both ensure the results are robust and also protect us from ad-hoc analyses proposed by potential reviewers. We have already benefited from the use of preregistration prior to submitting our manuscript for peer review. Specifically, during the data analysis meeting, it was proposed that age might further moderate the neuroticism by conscientiousness interaction. Regardless of the theoretical merits of this idea, it is ill-advised to design new analyses after seeing the results, as this violates the assumptions of null hypothesis significance testing (Weston et al. 2019b). Rather than simply throw out these analyses, we were able to add them to the project (efficiently, thanks to the use of scripting software) and present these analyses under the header of Unregistered Analyses. That is, we were not precluded from performing any analyses, allowing for the examination of any potentially important effect; however, we avoided problematic interpretations of these analyses by clearly labeling them as exploratory. (There was no evidence of this three-way interaction.) 


\section{Consider generalizability}

The choice of datasets informed our ability to estimate generalizability. One especially important factor - the measurement instrument used to assess neuroticism - was diversely sampled, as across these 15 datasets, we were able to compare results using five common personality scales: the NEO-FFI (Costa and McCrae, 1989), the NEO-PI-R (Costa and McCrae. 2008), the BFI (joh), the MIDI (Lachman and Weaver 1997), and (Goldberg et al., 2006, 's) 50 Adjectives. In other ways, however, our generalizability was limited. All studies came from mainly white, industrialized (American, European or Australian) countries, the majority of participants were over the age of 55, and our measures were almost exclusively self-report (with the obvious exception of mortality).

In short, while a few analysts found statistically significant interactions of neuroticism and conscientiousness on some health behaviors and outcomes, overall, there was fairly consistent evidence that the interaction effect size did not differ from 0 (Turiano et al., 2019, Weston et al., 2019a). The primary exceptions to this was when conscientiousness was low, neuroticism was associated with more smoking and less activity; when conscientiousness is high, neuroticism was not associated with health behaviors (Graham et al., 2019b). We found no evidence that the use of one particular scale changed either the relationship of neuroticism to health, the relationship of conscientiousness to health, or the interaction. The ability to examine the role of specific personality scales was an important contribution of our coordinated analysis. An important note about the results of these coordinated analysis: the interaction of conscientiousness and neuroticism predicting smoking and physical activity was only statistically significant in the overall meta-analysis and within only two or three datasets. A primary benefit of coordinated analysis is the opportunity to put these effect sizes into context: there was no heterogeneity in the size of the effects and, for both analyses, the effect sizes were quite small. Taken together with the significant interactions, we can propose that the moderation effect is present but quite small. We would have been less certain in this statement if we had run a single study and been unable to determine whether the effect size was unusual.

\section{Summary of healthy neuroticism coordinated analysis}

This is an example of when coordinated analysis is an appropriate and useful tool for research. The research question in this study - whether the interaction of neuroticism and conscientiousness associated with health - is both simple and yet in-adequately answered by the published literature. We were able to assemble a relatively large number of panel studies to test this question using several different outcomes. We concluded that relatively few studies yielded a significant interaction, that the weighted average effect across all studies was null, and that this effect size was not further moderated by personality scale or age. Moreover, by using scripting technology, we were able to efficiently ensure that no additional variability was added to our study, quickly synthesize results, spark discussion among a large team of analysts and researchers, and foster a hands-on learning experience for junior researchers. Through preregistration, we were able to ensure the robust and careful interpretation of our results.

\section{Conclusion}

Coordinated analysis is one method of facilitating robust results using pre-existing data. Coordinated analysis involves the estimation of a simple relationship or model in multiple pre-existing datasets and then using these estimates to create an overall weighted effect size or model. With the improvement of open source scripting technology, coordinated analysis becomes easier, even across large teams of analysts. Scripting also allows for integration of results into manuscripts with minimal human error. Coordinated analysis can be used with other Open Science tools like preregistration to ensure the responsible interpretation of results. Finally, by paying attention to differences across datasets in location, time, measurement instruments and sample characteristics, the generalizability of results can be estimated as well.

The analysis of pre-existing data is difficult, especially as researchers return to the same datasets multiple times, and by becoming aware of relationships between study variables are unable to unambiguously interpret significance tests with one or even a couple datasets. Despite these issues, lifespan researchers must continue to use pre-existing datasets, as they are often the best tools for answering important lifespan research questions. The use of coordinated analysis can address the potential analytic problems that come with pre-existing data, by leveraging the availability of large datasets to answer simple but important research questions, limiting researcher degrees of freedom, and testing moderators imposed by time, location and study design. 


\section{References}

Atherton, O. E., Robins, R. W., Rentfrow, P. J., and Lamb, M. E. (2014). Personality correlates of risky health outcomes: Findings from a large Internet study. Journal of Research in Personality, 50:56 60.

Baltes, P. B. and Nesselroade, J. R. (1979). Longitudinal research in the study of behavior and development, volume 10. Academic Press New York, NY.

Brim, O. G., Baltes, P. B., Bumpass, L. L., Cleary, P. D., Featherman, D. L., Hazzard, W. R., and Shweder, R. (1999). Midlife in the united states (midus 1), 1995-1996. Ann Arbor, MI: Inter-university Consortium for Political and Social Research. https://doi. org/https://doi. org/10.3886/ICPSR02760. v12.

Costa, P. and McCrae, R. (1989). Neo five-factor inventory (neo-ffi). Odessa, FL: Psychological Assessment Resources, 3.

Costa, P. T. and McCrae, R. R. (2008). The revised neo personality inventory (neo-pi-r). The SAGE handbook of personality theory and assessment, 2(2):179-198.

Crippa, A., Khudyakov, P., Wang, M., Orsini, N., and Spiegelman, D. (2016). A new measure of between-studies heterogeneity in meta-analysis. Statistics in medicine, 35(21):3661-3675.

Elliot, A. J., Turiano, N. A., and Chapman, B. P. (2016). Socioeconomic status interacts with conscientiousness and neuroticism to predict circulating concentrations of inflammatory markers. Annals of Behavioral Medicine, 51(2):240-250.

Friedman, H. S. (2000). Long-term relations of personality and health: Dynamisms, mechanisms, tropisms. Journal of personality, 68(6):1089-1107.

Friedman, H. S. (2019). Neuroticism and health as individuals age. Personality Disorders: Theory, Research, and Treatment, 10(1):25-32.

Friedman, H. S., Kern, M. L., and Reynolds, C. A. (2010). Personality and Health, Subjective Well-Being, and Longevity. Journal of Personality, 78:179-216.

Gale, C. R., Čukić, I., Batty, G. D., McIntosh, A. M., Weiss, A., and Deary, I. J. (2017). When is higher neuroticism protective against death? findings from uk biobank. Psychological science, 28(9):1345-1357.

Gelman, A. and Loken, E. (2013). The garden of forking paths: Why multiple comparisons can be a problem, even when there is no "fishing expedition" or "p-hacking" and the research hypothesis was posited ahead of time. Department of Statistics, Columbia University.

Goldberg, L. R., Johnson, J. A., Eber, H. W., Hogan, R., Ashton, M. C., Cloninger, C. R., and Gough, H. G. (2006). The international personality item pool and the future of public-domain personality measures. Journal of Research in personality, 40(1):84-96.

Graham, E. K., Weston, S. J., Gerstorf, D., Yoneda, T. B., Booth, T., Beam, C. R., Petkus, A. J., Rutsohn, J. P., Drewelies, J., Hall, A. N., Bastarache, E. D., Estabrook, R., Katz, M. J., Turiano, N. A., Lindenberger, U., Smith, J., Wagner, G. G., Pedersen, N. L., Allemand, M., Spiro III, A., Deeg, D. J. H., Johansson, B., Piccinin, A. M., Sliwinski, M., Lipton, R. B., Schaie, K. W., Willis, S., Reynolds, C. R., Deary, I. J., Hofer, S. M., and Mroczek, D. K. (2019a). A coordinated analysis of big-five trait change across 16 longitudinal studies. under review.

Graham, E. K., Weston, S. J., Turiano, N. A., Booth, T., Harrison, F., James, B. D., Mroczek, D. K., et al. (2019b). Is healthy neuroticism associated with health?: A coordinated integrative data analysis. under review.

Hagger-Johnson, G., Roberts, B., Boniface, D., Sabia, S., Batty, G. D., Elbaz, A., Singh-Manoux, A., and Deary, I. J. (2012). Neuroticism and cardiovascular disease mortality: socioeconomic status modifies the risk in women (uk health and lifestyle survey). Psychosomatic medicine, 74(6):596-603.

Hampson, S. E. and Goldberg, L. R. (2006). A first large cohort study of personality trait stability over the 40 years between elementary school and midlife. Journal of personality and social psychology, 91(4):763.

Higgins, J. P. and Thompson, S. G. (2002). Quantifying heterogeneity in a meta-analysis. Statistics in medicine, 21(11):1539-1558.

Hill, P. L., Turiano, N. A., Mroczek, D. K., and Burrow, A. L. (2016). The value of a purposeful life: Sense of purpose predicts greater income and net worth. Journal of research in personality, 65:38-42.

Hofer, S. M. and Piccinin, A. M. (2009). Integrative data analysis through coordination of measurement and analysis protocol across independent longitudinal studies. Psychological methods, 14(2):150-164. 
Hofer, S. M. and Piccinin, A. M. (2010). Toward an integrative science of life-span development and aging. Journals of Gerontology Series B: Psychological Sciences and Social Sciences, 65(3):269-278.

Lachman, M. E. and Weaver, S. L. (1997). The midlife development inventory (midi) personality scales: Scale construction and scoring. Waltham, MA: Brandeis University, pages 1-9.

Lalkhen, A. G. and McCluskey, A. (2008). Statistics v: Introduction to clinical trials and systematic reviews. Continuing Education in Anaesthesia, Critical Care \& Pain, 8(4):143-146.

McClelland, G. H. and Judd, C. M. (1993). Statistical difficulties of detecting interactions and moderator effects. Psychological bulletin, 114(2):376.

Munafò, M. R., Nosek, B. A., Bishop, D. V., Button, K. S., Chambers, C. D., Du Sert, N. P., Simonsohn, U., Wagenmakers, E.-J., Ware, J. J., and Ioannidis, J. P. (2017). A manifesto for reproducible science. Nature human behaviour, 1(1):0021.

Murray, A. L. and Booth, T. (2015). Personality and physical health. Current Opinion in Psychology, 5:50 - 55. Health behavior.

Piccinin, A. M. and Hofer, S. M. (2008). Integrative analysis of longitudinal studies on aging: Collaborative research networks, meta-analysis, and optimizing future studies. In Hofer, S. and Alwin, D., editors, Handbook on Cognitive Aging: Interdisciplinary Perspectives, pages 446-476. Sage, Thousand Oaks, CA.

Piccinin, A. M., Muniz-Terrera, G., Clouston, S., Reynolds, C. A., Thorvaldsson, V., Deary, I. J., Deeg, D. J., Johansson, B., Mackinnon, A., Spiro III, A., et al. (2013). Coordinated analysis of age, sex, and education effects on change in mmse scores. Journals of Gerontology Series B: Psychological Sciences and Social Sciences, 68(3):374-390.

Shipley, B. A., Weiss, A., Der, G., Taylor, M. D., and Deary, I. J. (2007). Neuroticism, extraversion, and mortality in the uk health and lifestyle survey: a 21-year prospective cohort study. Psychosomatic Medicine, 69(9):923-931.

Simmons, J. P., Nelson, L. D., and Simonsohn, U. (2011). False-positive psychology: Undisclosed flexibility in data collection and analysis allows presenting anything as significant. Psychological science, 22(11):1359-1366.

Stephan, Y., Sutin, A. R., Canada, B., and Terracciano, A. (2017). Personality and frailty: Evidence from four samples. Journal of research in personality, 66:46-53.

Sudlow, C., Gallacher, J., Allen, N., Beral, V., Burton, P., Danesh, J., Downey, P., Elliott, P., Green, J., Landray, M., et al. (2015). Uk biobank: an open access resource for identifying the causes of a wide range of complex diseases of middle and old age. PLoS medicine, 12(3):e1001779.

Turiano, N. A., Graham, E. K., Weston, S. J., Booth, T., Harrison, F., James, B. D., Mroczek, D. K., et al. (2019). Is healthy neuroticism associated with longevity?: A coordinated integrative data analysis. under review.

Turiano, N. A., Mroczek, D. K., Moynihan, J., and Chapman, B. P. (2013). Big 5 personality traits and interleukin-6: Evidence for "healthy neuroticism" in a us population sample. Brain, behavior, and immunity, 28:83-89.

Turiano, N. A., Whiteman, S. D., Hampson, S. E., Roberts, B. W., and Mroczek, D. K. (2012). Personality and substance use in midlife: Conscientiousness as a moderator and the effects of trait change. Journal of Research in Personality, 46(3):295 - 305.

Vartanian, T. P. (2010). Secondary data analysis. Oxford University Press.

Vollrath, M. and Torgersen, S. (2002). Who takes health risks? a probe into eight personality types. Personality and Individual Differences, 32(7):1185 - 1197.

Weston, S. J., Graham, E. K., Turiano, N. A., Aschwanden, D., Booth, T., Harrison, F., James, B. D., Mroczek, D. K., et al. (2019a). Is healthy neuroticism associated with chronic conditions?: A coordinated integrative data analysis. under review.

Weston, S. J., Hill, P. L., Edmonds, G. W., Mroczek, D. K., and Hampson, S. E. (2018). No evidence of "healthy neuroticism" in the hawaii personality and health cohort. Annals of Behavioral Medicine, 53(5):426-441.

Weston, S. J. and Jackson, J. J. (2015). Identification of the healthy neurotic: Personality traits predict smoking after disease onset. Journal of Research in Personality, 54:61 - 69. R Special Issue.

Weston, S. J. and Jackson, J. J. (2016). How do people respond to health news? The role of personality traits. Psychology \& Health, 31:1-18.

Weston, S. J., Ritchie, S. J., Rohrer, J. M., and Przybylski, A. K. (2019b). Recommendations for increasing the transparency of analysis of pre-existing datasets. Advanced Methods and Practices in Psychological Science.

Yarkoni, T. and Westfall, J. (2017). Choosing prediction over explanation in psychology: Lessons from machine learning. Perspectives on Psychological Science, 12(6):1100-1122. 